\title{
Combinação de métodos paramétricos e não-paramétricos na análise de estabilidade de cultivares de cana-de-açúcar ${ }^{(1)}$
}

\author{
Norberto Antonio Lavorenti(2) e Sizuo Matsuoka(3)
}

\begin{abstract}
Resumo - A presença e a magnitude da interação genótipo-ambiente são fatores que determinam o desempenho de cultivares nos diversos ambientes de produção. A forma de teste e interpretação dessa interação tem sido muito discutida na literatura agronômica, e vários métodos têm sido propostos. $\mathrm{O}$ objetivo deste trabalho foi testar uma combinação de métodos paramétricos e não-paramétricos para avaliação da interação genótipo-ambiente de forma simples e fácil. Foram utilizados os resultados experimentais de três grupos de cultivares de cana-de-açúcar, de acordo com suas características de maturação (precoce, média e tardia), em média de três cortes, em cinco locais da região Oeste do Estado de São Paulo. Os testes utilizados, além de simples, permitiram indicar as melhores cultivares para aquela região e para cada época de colheita, e confirmaram, através de modelos probabilísticos, as recomendações anteriormente realizadas pelos melhoristas.
\end{abstract}

Termos para indexação: Saccharum officinarum, interação genótipo-ambiente, métodos estatísticos.

\section{Combination parametric and non-parametric methods for stability analysis of sugarcane cultivars}

Abstract - The presence and magnitude of the interaction genotype-environment are factors that determine the performance of cultivars in distinct environments of sugarcane production. Methods for testing and interpreting that interaction have been a matter of concern in the agronomic literature, and several methods have been proposed. In this work the feasibility of using a combination of parametric and nonparametric statistical methods was tested for evaluating that interaction. Results of three successive crops in five locations, of Western São Paulo State, Brazil, and in three groups of experiments comprising sugarcane cultivars of similar maturation (early, intermediate and late) characteristics in each, were used as basic data. It was concluded that the method evaluated, besides its simplicity, permitted the identification of cultivars with better stability for the different kinds of environments tested, confirming previous recommendations of the breeders.

Index terms: Saccharum officinarum, genotype-environment interaction, statistical methods.

\section{Introdução}

O nível da interação genótipo-ambiente em qualquer nova cultivar há que ser medido por todo melhorista antes de se recomendá-la aos agricultores. A estes, os riscos na adoção de uma cultivar serão tanto menores quanto maior for a adaptabili-

\footnotetext{
(1) Aceito para publicação em 28 de julho de 2000 .

(2) Universidade Federal de São Carlos (UFSCar), Centro de Ciências Agrárias, Dep. de Tecnologia Agroindustrial e Sócio-economia Rural, Caixa Postal 153, CEP 13600-970 Araras, SP. E-mail: norba@cca.ufscar.br

(3) UFSCar, CCA, Dep. de Biotecnologia Vegetal. E-mail: sizuo@dbv.cca.ufscar.br
}

dade da cultivar aos ambientes específicos de sua propriedade, como também quanto maior for a sua capacidade homeostática a variações imprevisíveis (Lin \& Binns, 1988). Homeostase é a capacidade da planta em adaptar suas funções fisiológicas às constantes mudanças do ambiente onde ela cresce, de forma a ser menos afetada por elas. Estabilidade é o termo que define tecnicamente tal condição (Breese, 1969). Ainda que não haja unanimidade na conceituação desses termos, e muito menos nos diversos métodos estatísticos propostos para suas determinações (Finlay \& Wilkinson, 1963; Eberhart \& Russel, 1966; Verma et al., 1978; Silva \& Barreto, 1985; Lin et al., 1986; Lin \& Binns, 1988; Cruz et al., 1989; Chakroun et al., 1990; Evans, 1996), ao agricul- 
tor é importante a conjugação da adaptabilidade com a estabilidade, isto é, que a cultivar seja confiável o suficiente para substituir outra já conhecida.

A determinação da estabilidade não é uma questão simples. As cultivares variam muito nas suas capacidades de tamponamento ao ambiente, e este possui muitos componentes variáveis e imprevisíveis, resultantes da conjugação dos fatores do solo e do clima, em interação com diversos elementos externos, de ação antrópica ou não, inclusive a ocorrência de doenças e pragas. Por essa razão, resultam muitas dificuldades para a devida avaliação de cultivares. E essas dificuldades são maiores no caso da cana-de-açúcar, cuja exploração é de bastante complexidade. Uma grande dificuldade neste caso é a sua característica de semiperenidade, com a produção sendo medida na mesma parcela por vários anos e, assim, a cada ano ocorrer uma diferente condição de reinício da cultura (Chakroun et al., 1990). Além disso, como a safra se estende por muitos meses, a colheita ocorre em pelo menos três distintas estações do ano no caso da região centro-sul: outono, inverno e primavera. Isso implica na necessidade de cultivares de distintas capacidades de maturação, para que se possa efetuar colheita escalonada durante a safra. Uma das conseqüências disso é que, de acordo com a época de colheita, cada cultivar sofrerá, a cada ano, influência marcante e errática, inicialmente pelo deslocamento das máquinas sobre os estolhos em distintas condições de umidade no solo, e, logo a seguir, pela diferenciada seqüência climática para o mesmo estádio fenológico. A semiperenidade traz ainda uma séria implicação para o agricultor: adotada a cultivar, esta terá que ser cultivada por vários anos. Ela deverá, portanto, ser a mais confiável possível. Para tanto, há necessidade de testes e experimentação nas mais variadas condições de cultivo e, posteriormente, métodos de análise que permitam a discriminação apropriada.

O objetivo deste trabalho foi testar uma combinação de métodos paramétricos e não-paramétricos para avaliação da interação genótipo-ambiente de forma simples e fácil.

\section{Material e Métodos}

Os experimentos objeto desta análise abrangeram três grupos de genótipos de cana-de-açúcar, de acordo com suas características de maturação previamente conhecidas, isto é, precoces, médias e tardias (Copersucar, 1991; Arizono et al., 1994; Universidade Federal de São Carlos, 1994, 1996; Matsuoka et al., 1995, 1998). As análises estatísticas foram realizadas independentemente em cada conjunto de cultivares, de acordo com os agrupamentos mencionados.

Os experimentos foram instalados entre março e abril de 1994, e colhidos sucessivamente por três safras. Eles foram plantados em cinco propriedades da região Oeste do Estado de São Paulo (destilarias Álcool Azul, Centrálcool, Generalco, Unialco e Vale do Rio Turvo), cujo clima é classificado como tropical quente subseco, e onde, de acordo com Dias (1997) e Dias et al. (1999), predominam argissolos, mas também ocorrem latossolos (Landell et al., 1997). Somente foram consideradas na análise as cultivares de cada época de maturação que estavam presentes em todos os 14 ambientes (cortes x locais). O terceiro corte de um experimento foi perdido por queima acidental. A variável tonelada de pol por hectare (TPH) foi a base utilizada para os procedimentos estatísticos.

\section{Medidas paramétricas de estabilidade}

A medida paramétrica mais direta para avaliar estabilidade é a variância que mede a dispersão da média individual de uma cultivar em relação à média desta cultivar em diversos ambientes. Intimamente relacionado com essa medida está o coeficiente de variação de cada cultivar (Francis \& Kannemberg, 1978). Aqueles genótipos que têm pequena variância e pequeno coeficiente de variação são considerados estáveis, isto é, não existem grandes variações nas respostas de uma cultivar de um ambiente para outro.

Para enfatizar o comportamento de cada cultivar em cada local foi estruturado um intervalo de confiança, ao nível de confiança de $95 \%$, utilizando a média de cada ambiente $\mathrm{j}\left(\overline{\mathrm{x}}_{\mathrm{j}}\right)$ mais ou menos, aproximadamente, duas vezes o erro-padrão da média $\left(\mathrm{s}_{\mathrm{j}}\left(\overline{\mathrm{x}}_{\mathrm{j}}\right)\right)$, em cada local. Este intervalo foi construído da seguinte forma: a partir da análise de variância individual em cada ambiente ( $\mathrm{k}$ cultivares em $r$ blocos) estima-se o erro-padrão das médias das cultivares, ou seja:

$$
\mathrm{s}_{\mathrm{j}}(\overline{\mathrm{x}})_{\mathrm{j}}=\frac{\sqrt{\mathrm{QMResíduo}_{(\mathrm{j})}}}{\sqrt{\mathrm{r}}}
$$

onde,

QM Resíduo $_{(j)}$ : quadrado médio do resíduo ou variância ambiental do ambiente j;

r: número de blocos. 
Assim, estrutura-se:

I.C. $(95 \%) \Rightarrow \bar{x}_{j} \pm 2 s_{j}\left(\bar{x}_{j}\right)$,

estabelecendo-se assim os limites inferior e superior do intervalo de confiança.

Atribuiu-se o sinal negativo à cultivar cuja média estivesse abaixo do limite inferior do intervalo de confiança, e, por outro lado, o sinal positivo àquela que estivesse com a média acima do limite superior do intervalo de confiança; o valor zero foi atribuído à média da cultivar contida entre aqueles limites.

Em todos os ambientes foi feita a contagem do número de sinais positivos, negativos e nulos. Cultivares com grande diversidade de sinais positivos e negativos indicam uma forte interação genótipo-ambiente, não sendo normalmente recomendadas para programas de melhoramento, devido à sua baixa estabilidade, mas podendo eventualmente ser de interesse para ambientes específicos.

Cultivares com uma concentração de sinais negativos e nulos indicam estabilidade, mas não seriam selecionáveis devido à sua baixa produtividade. Por outro lado, aquelas com uma concentração de sinais positivos seriam consideradas estáveis e, principalmente, teriam características desejáveis do ponto de vista do melhoramento genético.

\section{Medidas não-paramétricas de estabilidade}

Na classificação das médias de produção, dentro de um mesmo ambiente, o posto 1 foi atribuído à cultivar de maior produção média e o posto $\mathrm{p}$, à cultivar de menor produção média. Caso houvesse empate nos postos atribuía-se a média aritmética dos postos às cultivares envolvidas.

Huehn (1990) apresentou a medida mais simples de estabilidade no campo não-paramétrico, a qual se baseia na variância dos postos para uma determinada cultivar, ao longo de todos os ambientes. Ele obteve essa estimativa pela seguinte equação:

$S_{i}^{(2)}=\frac{\sum_{j=1}^{n}\left(r_{i j}-\bar{r}_{i}\right)^{2}}{(n-1)}$

onde:

$\mathrm{S}_{\mathrm{i}}^{(2)}$ é a variância de cada cultivar i baseada nos postos de todos os ambientes;

$\mathrm{r}_{\mathrm{ij}}$ é o posto (classificação) da cultivar i no ambiente $\mathrm{j}$;

$\overline{\mathrm{r}}_{\mathrm{i}}$ é a média dos postos da cultivar i nos $\mathrm{n}$ ambientes.

Portanto, as cultivares que apresentassem as menores variâncias nos postos foram consideradas as mais estáveis. Fox et al. (1990) propuseram uma estratificação des- tes postos para a definição de estabilidade de determinada cultivar. Eles sugeriram, de maneira mais intuitiva, que as cultivares cujas produções ocupassem consistentemente o terço superior dos postos (menores valores $=$ maiores produções) seriam estáveis e teriam características favoráveis à seleção, ao passo que aquelas que ocupassem o terço inferior dos postos (maiores valores $=$ menores produções) seriam igualmente estáveis, mas não seriam selecionáveis, devido à baixa produção.

O que se visualizou com este trabalho foi propor uma combinação dos dois métodos, ou seja, todas as cultivares, em cada época de maturação, que simultaneamente fossem selecionadas pelo método do Intervalo de Confiança (95\%) e pelo método não-paramétrico proposto por Fox et al. (1990), seriam as mais recomendadas, pois teriam não somente alta produção mas também a desejada estabilidade.

\section{Resultados e Discussão}

Na Tabela 1 estão apresentadas as médias e as variâncias das 11 cultivares precoces presentes nos 14 ambientes e um resumo da quantidade de sinais positivos, negativos e nulos, ao longo de todos os ambientes. $\mathrm{O}$ acúmulo de sinais positivos em uma cultivar indica que ela se sobressaiu em relação às demais cultivares na maioria dos ambientes, com produção superior à das demais em cada um dos ambientes, denotando, assim, maior estabilidade.

No caso de uma cultivar apresentar sinais positivos e negativos, ter-se-ia clara indicação de ausência de estabilidade, ou seja, que estaria ocorrendo alta interação genótipo-ambiente, o que dificultaria, por exemplo, uma recomendação regional. No entanto, poderia ser recomendada numa condição específica, para tanto exigindo confirmação quanto ao exato ambiente de adaptação. Outro caso de estabilidade seria aquele obtido com um acúmulo de sinais negativos, o que, porém, indicaria baixa competitividade com os demais genótipos.

De acordo com os resultados paramétricos puderam ser selecionadas as cultivares de maturação precoce RB835486 e RB855035, que seriam consideradas estáveis e com alta produtividade. Seriam descartadas RB765418, RB855156, RB855574 e SP78-5495, sendo que as demais poderiam ser cultivadas se consideradas vantagens específicas.

O uso do método não-paramétrico proposto neste trabalho permite a seleção das cultivares de alta estabilidade (terço superior da variância dos índi- 
Tabela 1. Combinação de métodos estatísticos para determinação da estabilidade de cultivares de cana-de-açúcar de maturação precoce na região Oeste do Estado de São Paulo.

\begin{tabular}{|c|c|c|c|c|c|c|c|c|c|}
\hline \multirow[t]{3}{*}{ Cultivar } & \multicolumn{5}{|c|}{ Método paramétrico } & \multicolumn{4}{|c|}{ Método não-paramétrico } \\
\hline & \multirow{2}{*}{$\begin{array}{c}\text { Média } \\
\text { geral }\end{array}$} & \multirow{2}{*}{$\begin{array}{c}\text { Variância } \\
\text { geral }\end{array}$} & \multirow{2}{*}{$\begin{array}{c}\text { Totais } \\
(-)\end{array}$} & \multirow{2}{*}{$\begin{array}{c}\text { Totais } \\
(0)\end{array}$} & \multirow{2}{*}{$\begin{array}{c}\text { Totais } \\
(+)\end{array}$} & \multirow{2}{*}{$\begin{array}{l}\text { Média dos } \\
\text { índices }\end{array}$} & \multirow{2}{*}{$\begin{array}{c}\text { Variância dos } \\
\text { índices }\end{array}$} & \multicolumn{2}{|c|}{ Terço superior } \\
\hline & & & & & & & & Média & Variância \\
\hline RB765418 & 13,33 & 7,67 & 8 & 6 & 0 & 9,07 & 6,99 & - & - \\
\hline RB825317 & 15,61 & 17,55 & 1 & 13 & 0 & 6,36 & 6,55 & 0 & - \\
\hline RB825336 & 16,60 & 13,63 & 0 & 14 & 0 & 5,14 & 4,13 & 0 & + \\
\hline RB835486 & 18,63 & 11,21 & 0 & 7 & 7 & 1,93 & 1,61 & + & + \\
\hline RB855035 & 18,81 & 10,09 & 0 & 6 & 8 & 2,43 & 2,57 & + & + \\
\hline RB855046 & 15,52 & 8,00 & 0 & 14 & 0 & 6,86 & 5,52 & 0 & - \\
\hline RB855156 & 15,40 & 14,77 & 3 & 11 & 0 & 6,86 & 8,29 & 0 & - \\
\hline RB855206 & 16,31 & 12,73 & 0 & 14 & 0 & 4,86 & 4,75 & 0 & 0 \\
\hline RB855563 & 16,67 & 9,95 & 0 & 12 & 2 & 5,00 & 4,92 & 0 & 0 \\
\hline RB855574 & 14,65 & 11,20 & 3 & 11 & 0 & 8,00 & 4,62 & - & 0 \\
\hline SP78-5495 & 13,58 & 17,17 & 6 & 8 & 0 & 9,50 & 4,27 & - & 0 \\
\hline
\end{tabular}

ces) e, simultaneamente, alta produção (terço superior da média dos índices). Assim, seriam indicadas como precoces as mesmas cultivares RB835486 e RB855035 indicadas pelo método anterior. $\mathrm{Na}$ combinação dos dois processos estatísticos, portanto, aquelas duas cultivares precoces seriam recomendadas.

Para as cultivares de maturação média, 14 cultivares estavam sendo representadas nos 14 ambientes estudados, e os resultados relativos a TPH são os apresentados na Tabela 2 .

Utilizando os mesmos critérios adotados anteriormente, no campo paramétrico seriam selecionadas as cultivares RB835486, RB855035 e RB855536, que foram as que apresentaram a maior concentração de sinais positivos, mas também podendo ser recomendadas as cultivares RB72454 e RB855113. Por outro lado, no campo não-paramétrico seriam selecionadas as cultivares RB835486 e RB855113. Portanto, em termos de maturação média, pela combinação dos dois métodos, pode-se recomendar a cultivar RB835486 como a mais indicada para aquela região.

A cultivar RB855035 obteve as primeiras colocações em praticamente todos os ambientes, exceto nos três cortes da destilaria Generalco, onde foi a undécima colocada no primeiro corte e a décima terceira nos dois cortes seguintes. Isto fez com que a variabilidade dos índices subisse muito. Talvez esta cultivar merecesse um estudo mais detalhado no tipo de solo desse local.

Somente sete cultivares de maturação tardia estiveram presentes nos 14 ambientes estudados, e os resultados dos métodos estatísticos empregados estão apresentados na Tabela 3.

Neste grupo de cultivares, dentro dos critérios estabelecidos, não foram observadas cultivares que satisfizessem os dois métodos propostos. Houve somente indicações de algumas, denominadas de potencialmente selecionáveis. Assim, no campo paramétrico poder-se-ia indicar as cultivares RB72454, RB845257 e RB855113 como sendo as mais promissoras, ao passo que no campo não-paramétrico seriam indicadas RB72454 e RB845257. Portanto, na combinação dos dois métodos, as cultivares que potencialmente atenderiam as exigências da região como tardias seriam a RB72454 e a RB845257.

As cultivares assinaladas quanto às três épocas de maturação seriam as de maior confiabilidade em termos das condições médias do ambiente da região, a não ser que alguma particularidade venha a ser impeditiva. O estudo mostrou que existiram outras cultivares estáveis selecionáveis (da mesma forma que estáveis não-selecionáveis), mas que em termos comparativos de produtividade foram inferiores. Eventualmente uma ou outra poderá ter alguma vantagem competitiva em condições específicas. 
Tabela 2. Combinação de métodos estatísticos para determinação da estabilidade de cultivares de cana-de-açúcar de maturação média na região Oeste do Estado de São Paulo.

\begin{tabular}{|c|c|c|c|c|c|c|c|c|c|}
\hline \multirow[t]{3}{*}{ Cultivar } & \multicolumn{5}{|c|}{ Método paramétrico } & \multicolumn{4}{|c|}{ Método não-paramétrico } \\
\hline & \multirow{2}{*}{$\begin{array}{c}\text { Média } \\
\text { geral }\end{array}$} & \multirow{2}{*}{$\begin{array}{c}\text { Variância } \\
\text { geral }\end{array}$} & \multirow{2}{*}{$\begin{array}{c}\text { Totais } \\
(-)\end{array}$} & \multirow{2}{*}{$\begin{array}{c}\text { Totais } \\
(0)\end{array}$} & \multirow{2}{*}{$\begin{array}{c}\text { Totais } \\
(+)\end{array}$} & \multirow{2}{*}{$\begin{array}{c}\text { Média dos } \\
\text { índices }\end{array}$} & \multirow{2}{*}{$\begin{array}{c}\text { Variância dos } \\
\text { índices }\end{array}$} & \multicolumn{2}{|c|}{ Terço superior } \\
\hline & & & & & & & & Média & Variância \\
\hline RB72454 & 18,81 & 19,43 & 0 & 12 & 2 & 5,79 & 10,03 & + & 0 \\
\hline RB806043 & 15,38 & 17,13 & 5 & 9 & 0 & 12,21 & 1,87 & - & + \\
\hline RB825336 & 16,78 & 15,83 & 4 & 10 & 0 & 9,21 & 14,49 & - & - \\
\hline RB835089 & 18,08 & 18,32 & 1 & 13 & 0 & 6,57 & 12,11 & 0 & - \\
\hline RB835486 & 20,23 & 12,08 & 0 & 7 & 7 & 3,07 & 5,61 & + & + \\
\hline RB845197 & 17,21 & 17,25 & 0 & 14 & 0 & 8,43 & 5,65 & 0 & + \\
\hline RB845239 & 16,78 & 21,07 & 0 & 14 & 0 & 10,07 & 5,30 & - & + \\
\hline RB845257 & 18,15 & 32,61 & 2 & 11 & 1 & 6,57 & 17,19 & 0 & - \\
\hline RB845276 & 17,17 & 24,35 & 1 & 13 & 0 & 8,93 & 10,99 & 0 & 0 \\
\hline RB855035 & 19,24 & 12,03 & 2 & 6 & 6 & 4,57 & 19,49 & + & - \\
\hline RB855113 & 18,89 & 22,50 & 0 & 12 & 2 & 4,79 & 6,64 & + & + \\
\hline RB855536 & 19,35 & 22,09 & 0 & 9 & 5 & 5,43 & 16,26 & + & - \\
\hline RB855574 & 15,81 & 14,40 & 3 & 11 & 0 & 10,93 & 6,84 & - & 0 \\
\hline SP79-1011 & 17,31 & 21,29 & 0 & 14 & 0 & 8,43 & 13,65 & 0 & 0 \\
\hline
\end{tabular}

Tabela 3. Combinação de métodos estatísticos para determinação da estabilidade de cultivares de cana-de-açúcar de maturação tardia na região Oeste do Estado de São Paulo.

\begin{tabular}{|c|c|c|c|c|c|c|c|c|c|}
\hline \multirow[t]{3}{*}{ Cultivar } & \multicolumn{5}{|c|}{ Método paramétrico } & \multicolumn{4}{|c|}{ Método não-paramétrico } \\
\hline & \multirow{2}{*}{$\begin{array}{l}\text { Média } \\
\text { geral }\end{array}$} & \multirow{2}{*}{$\begin{array}{l}\text { Variância } \\
\text { geral }\end{array}$} & \multirow{2}{*}{$\begin{array}{c}\text { Totais } \\
(-)\end{array}$} & \multirow{2}{*}{$\begin{array}{l}\text { Totais } \\
(0)\end{array}$} & \multirow{2}{*}{$\begin{array}{l}\text { Totais } \\
(+)\end{array}$} & \multirow{2}{*}{$\begin{array}{l}\text { Média dos } \\
\text { índices }\end{array}$} & \multirow{2}{*}{$\begin{array}{l}\text { Variância dos } \\
\text { índices }\end{array}$} & \multicolumn{2}{|c|}{ Terço superior } \\
\hline & & & & & & & & Média & Variância \\
\hline RB82454 & 18,63 & 36,87 & 0 & 12 & 2 & 2,71 & 2,84 & + & 0 \\
\hline RB806043 & 15,26 & 26,09 & 2 & 12 & 0 & 6,29 & 0,84 & - & + \\
\hline RB815627 & 16,99 & 25,64 & 1 & 13 & 0 & 4,36 & 2,55 & 0 & 0 \\
\hline RB835089 & 17,12 & 40,44 & 1 & 13 & 0 & 4,43 & 3,65 & 0 & - \\
\hline RB845197 & 16,27 & 27,97 & 2 & 12 & 0 & 4,71 & 2,84 & 0 & - \\
\hline RB845257 & 18,91 & 38,87 & 0 & 12 & 2 & 2,36 & 2,55 & + & 0 \\
\hline RB855113 & 18,08 & 39,55 & 0 & 13 & 1 & 3,14 & 2,90 & + & - \\
\hline
\end{tabular}

O caso mais importante foi o da cultivar precoce RB855156 que, competitiva em alguns outros ambientes, não se comportou bem na região considerada. Para o agricultor, essa informação é tão importante quanto a de melhor adaptabilidade, pois muitas vezes ele é levado a plantar uma cultivar apenas por informações de seu bom comportamento em outras regiões, sem o devido teste no ambiente de sua propriedade.

Como o clima na região considerada não é fator marcante de determinação de comportamento varietal relativo (Dias, 1997; Dias et al., 1999), é importante que em cada propriedade seja feito um criterioso levantamento de solos, e, então, alocar as cultivares apropriadamente.

\section{Conclusões}

1. A combinação dos métodos estatísticos propostos possibilita boa discriminação de cultivares quanto à estabilidade. 
2. Os testes estatísticos utilizados são de grande simplicidade de aplicação.

\section{Referências}

ARIZONO, H.; GHELLER, A. C. A.; MASUDA, Y.; HOFFMANN, H. P.; BASSINELLO, A. I.; MATSUOKA, S. Opções de variedades RB de cana-deaçúcar. Álcool e Açúcar, São Paulo, n. 72, p. 26-31, fev./ mar. 1994.

BREESE, E. L. The measurement and significance of genotype-environment interaction in grasses. Heredity, Oxford, v. 24, p. 27-44, 1969.

CHAKROUN, M.; TALIAFERRO, C. M.; McNEW, R. W. Genotype-environment interactions of bermudagrass forage yields. Crop Science, Madison, v. 30, p. 49-53, 1990.

COPERSUCAR (São Paulo, SP). Terceira geração de variedades de cana Copersucar. São Paulo, 1991. 28 p. (Boletim Técnico Copersucar). Edição Especial.

CRUZ, C. D.; TORRES, R. A.; VENCOVSKY, R. An alternative approach to the stability analysis proposed by Silva and Barreto. Revista Brasileira de Genética, Ribeirão Preto, v. 12, n. 3, p. 567-580, 1989.

DIAS, F. L. F. Relação entre produtividade, clima, solos e variedades de cana-de-açúcar, na região noroeste do Estado de São Paulo. Piracicaba : ESALQ, 1997. 64 p. Dissertação de Mestrado.

DIAS, F. L. F.; MAZZA, J. A.; MATSUOKA, S.; PERECIN, D.; MAULLE, R. F. Produtividade da canade-açúcar em relação a clima e solos da região noroeste do Estado de São Paulo. Revista Brasileira de Ciência do Solo, Campinas, v. 23, n. 3, p. 627-634, 1999.

EBERHART, J. A.; RUSSEL, W. A. Stability parameters for comparing varieties. Crop Science, Madison, v. 6, p. 36-40, 1966.

EVANS, L. T. Crop evolution, adaptation and yield. Cambridge, Grã-Bretanha : Cambridge University Press, 1996. $500 \mathrm{p}$.

FINLAY, K. W.; WILKINSON, G. N. The analysis of adaptation in a plant breeding programme. Australian Journal of Agricultural Research, Collingwood, v. 14, p. 742-754, 1963.

FOX, P. N.; SKOUMAND, B.; THOMPSON, G. K.; BRAUN, H. J.; CORMIER, R. Yield and adaptation of hexaploid spring triticale. Euphytica, Dordrecht, v. 47, p. 57-64, 1990.
FRANCIS, T. R.; KANNEMBERG, L. W. Yield stability studies in short season maize. I. A descriptive method for grouping genotypes. Canadian Journal of Plant Science, Ottawa, v. 58, p. 1029-1034, 1978.

HUEHN, M. Nonparametric estimation and testing of genotype-by-environment interaction by ranks. In: KANG, M. S. (Ed.) Genotype-by-environment interaction and plant breeding. Baton Rouge : Lousiania State University Agricultural Center, 1990. p. 63-93.

LANDELL, M. G. A.; CAMPANA, M. P.; FIGUEIREDO, P.; ZIMBACK, L.; SILVA, M. A.; PRADO, $H$. Novas variedades de cana-de-açúcar. Campinas : Instituto Agronômico, 1997. 28 p. (IAC. Boletim Técnico, 169).

LIN, C. S.; BINNS, M. R. A method of analyzing cultivar x location x year experiments: a new stability parameter. Theoretical and Applied Genetics, Berlin, v. 76, p. 425430, 1988.

LIN, C. S.; BINNS, M. R.; LEFKOVITCH, L. P. Stability analysis. Where do we stand? Crop Science, Madison, v. 26 , p. 894-900, 1986.

MATSUOKA, S.; ARIZONO, H.; BASSINELLO, A.I.; GARCIA, A. A. F.; GHELLER, A. C. A.; GIGLIOTI, E.; HOFFMANN, H. P.; MASUDA, Y. Seis novas variedades RB de cana-de-açúcar. Araras : UFSCar, 1998. $24 \mathrm{p}$.

MATSUOKA, S.; ARIZONO, H.; BASSINELLO, A. I.; GHELLER, A. C. A.; HOFFMANN, H. P.; MASUDA, Y. Variedades superprecoces de cana-de-açúcar. Álcool e Açúcar, São Paulo, n. 78, p. 22-30, fev./mar. 1995.

SILVA, J. G.; BARRETO, J. N. Aplicação da regressão linear segmentada em estudos da interação genótipo por ambiente. In: SIMPÓSIO DE ESTATÍSTICA APLICADA À EXPERIMENTAÇÃO AGRONÔMICA, Piracicaba, 1985. Resumos... Piracicaba : Fundação Cargill, 1985. p. 40-50.

UNIVERSIDADE FEDERAL DE SÃO CARLOS. Centro de Ciências Agrárias. Departamento de Biotecnologia Vegetal. Programa de cooperação técnico-científica na área de melhoramento genético da cana-de-açúcar: relatório técnico 1994. Araras, 1994. 114 p.

UNIVERSIDADE FEDERAL DE SÃO CARLOS. Centro de Ciências Agrárias. Departamento de Biotecnologia Vegetal. Programa de cooperação técnico-científica na área de melhoramento genético da cana-de-açúcar: relatório técnico 1995. Araras, 1996. 94 p.

VERMA, M. M.; CHAHAL, G. S.; MURTY, B. R. Limitations of conventional regression analysis: a proposed modification. Theoretical and Applied Genetics, Berlin, v. 53, p. 89-91, 1978. 